\title{
A Monte Carlo Study of the Structure Forming of a Monolayer of Rod-like Molecules by a Modified Lattice Gas Model *
}

\author{
Fumitaka Matsubara and Yōichi KuroKawa ${ }^{* *}$ \\ Department of Engineering Science, Faculty of Engineering, \\ Tōhoku University, Aoba, Aramaki, Sendai 980
}

\section{Synopsis}

A modified lattice gas model for a monolayer of rod-like molecules has been proposed and investigated by using a Monte Carlo method. It has been shown that the monolayer is not uniform but consists of islands or aggregates.

Key words: Lattice gas model, Monolayer, Monte Carlo method
\end{abstract}

The relationship between the surface pressure and surface area of a monolayer has been investigated experimentally by many authors ${ }^{1)}$. The structure forming of the monolayer on the solution is a basic problem in surface chemistry. However, such a work has not been performed appreciably, since the exact structure of the monolayer in situ can be observed with great difficulty. A few information on the structure of the monolayer has already been provided by electron-microscopic studies of the monolayer transffered from a water surface ${ }^{2)}$. In certain cases, the monolayer is discontinuous and has a orientated structure. For a theoretical study of the isotherm of the monlayer on the surface, there are some works such as those based on a lattice gas model ${ }^{3,4}$. Baret et al. reported that the lattice gas model has been used well to describe the isother ${ }^{5)}$. However, it is not easy to show explicitly the structure forming of monolayer by using their model. Here, an attempt is made to study the structure forming of monolayer on

* 棒状分子の単分子膜構造形成に対する格子ガスモデルによ るモンテカルロ計算

** 松原史卓, 黒川洋一: 東北大学工学部基礎系教室, $\mathbf{\top} 980$ 仙台市荒巻字青葉 the basis of a modified lattice gas model by using a Monte Carlo method.

\section{Model}

We consider a gaseous monolayer of rod-like molecules on the surface of water. Taking into account a freedom of the direction of the molecule as well as a freedom of existence or non-existence of the molecule at each location, we propose the following simple model. We assume that the surface can be dealt as a two dimensional lattice and the molecules with their centers at lattice sites lie on bonds of the lattice. The length of the molecule and the structure of the lattice are arbitrary. Here, for simplicity, we assume that the lattice is of trianglar and the length is twice as long as the distance between nearestneighbor lattice sites, i. e., each molecule occupies three lattice sites. We further assume that, to exclude the superposition of molecules, each lattice site should not be occupied by more than one molecule. Interaction energies are allowed only between two molecules neighboring and parallel with each other such that they are proportional to contact length of the molecules. Hence, they take three distinct values $0,-V$, and $-3 \mathrm{~V}$ depending on the configuration of molecule 
I) $\frac{k T}{V}=1.2$
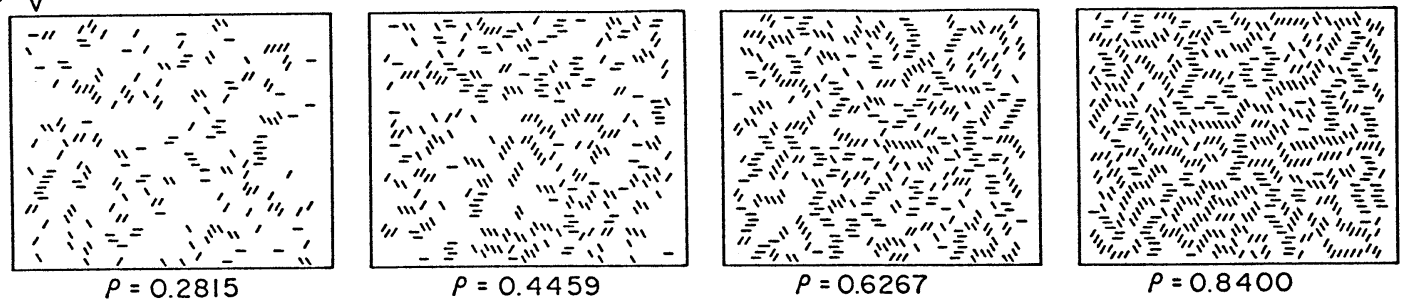

II) $\frac{k T}{V}=0.6$
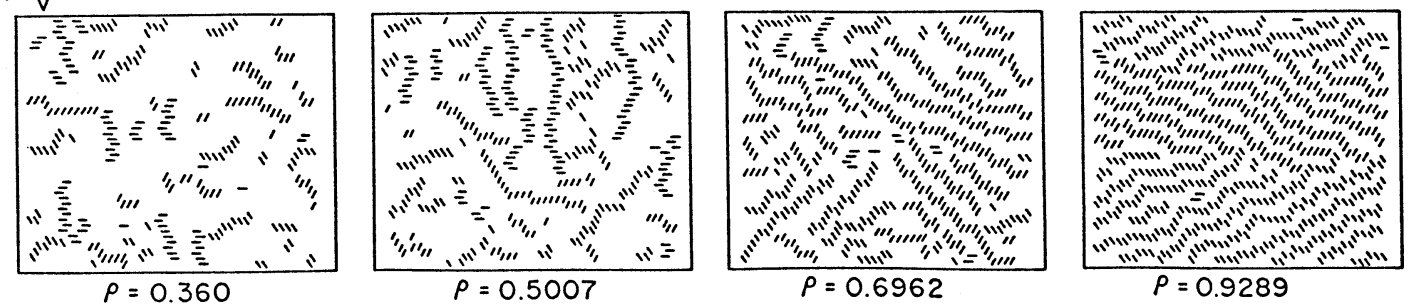

Fig. 1 Structure forming of a monolayer

on the lattice, where $-4 \mathrm{~V}$ is the energy between two fully contacted molecules. The chemical potential per molecule $\mu$ is also taken into account. We study the structure of the monolayer and the isotherm at various temperatures. To realize a thermally equilibrium configuration of molecules, we use a Monte Carlo method, where the probability of existence of molecule at each location is given according to the Gibbs distribution. Detailes of the procedure of the method will be described elsewhere.

\section{Results and Discussion}

Typical results of the structures of the molecules on the lattice are shown in Fig.1. Here, $T$, $k$ and $\rho$ are the temperature, Boltzmann constant, and the density of molecules defined by the number of the molecules per three lattice sites, respectively. On the whole, in lower densities, islands are formed and they are widely separated. As the density increases, the islands increase in length and in numbers. They are closely linked with each other but vacant spaces are observed among them. This behavior becomes more significant at low temperatures as shown in the figure. The islands are more closely linked and show string-like structure. In higher densities, the structure depends much more on the temperature. At low temperature, a few demains composed of strings are found. These strings align almost parallel with each other but they are not straight. On the other hand, at high temperature, we do not find the domains but see a packing of short strings and isolated molecules.

$R$ ies and Kimball presented an evidence that $n$ hexatriacontanoic acid monolayers are composed of island ${ }^{2)}$. As the pressure rises, these islands seem to join to form a continuous layer in which holes or vacant areas exist. The progressive reduction in these vacant areas leads finally to the formation of a close-packed monolayer. This behavior is analogous to that obtained in our model, though this behavior may not necessarily correspond to the case of our model because our model is the case for a gaseous monolayer.

Our model permits the calculations of various thermodynamic quantities, e.g. the relationship between the surface pressure and the density et al. These will be reported separately. We note that our model has a tendency that molecules take easily the same direction because of taking into account only three directions. We believe, 
however, that our model is useful to study the formation of the structure of rod-like molecules as the well-known lattice gas model is useful to study the isotherm of monolayer.

We have proposed a simple lattice gas model and treated it by using the Monte Carlo method. Many variations of our model can be easily made. Our model can be also extended to the monolayer of flexible long molecules, though the calculation is troublesome. For the monolayer of amphiphile molecules, the situation may be more complicated than this approach implies. However, such observation of monolayer islands as shown in this communication may provide information basic to two-dimensional crystallization, nucleation and micell formation.

\section{References}

1) G. L. Gains Jr.: "Insoluble Monolayers at Liquid-Gas Interface" Interscience Publishers (1966).

2) H.E. Ries Jr, W.A. Kimball: J. Phys. Chem., 59, 94, 992 (1955) ; H.E. Ries Jr, H. S. Swift: J. Colloid Inter. Sci. 64, 111 (1978); H. E. Ries Jr, M. Matsumoto, N. Ueda, E. Suito: ibid, 57, 396 (1976).

3) S. Ohki: J. Theoret. Biol, 15, 346 (1967).

4) A. Calle, A. Rapini, M.J.Zuckermann, A. Cros, S. Doniach: Can. J. Phys. 56, 348 (1978).

5) J-R. Baret, J-L. Firpo: J. Colloid Inter. Sci., 94, 487 (1983). 